\title{
Valutazione dello stato nutrizionale con l'uso dell'impedenziometria
}

\author{
D. Zazzaro, A. Sturniolo, G. Piccinni, G. Splendiani \\ Cattedra di Nefrologia, Università Tor Vergata, Roma
}

$\mathrm{n}$ un soggetto normale in caso di alterazione dello stato di benessere clinico (inteso come l'assenza di disturbi soggettivi ed oggettivi che alterino le normali condizioni di vita del paziente) è importante poter valutare lo stato di idratazione e di nutrizione per apportarne le eventuali correzioni.

Infatti, la mancanza di benessere clinico può essere correlato ad alterazioni dello stato nutrizionale e/o dello stato di idratazione e non ad una malattia vera e propria. Nella Tabella I sono definiti gli stati nutrizionali. Cattive abitudini alimentari possono portare ad uno stato di sovrappeso o di sottopeso: per quanto riguarda lo stato di idratazione, in genere $\mathrm{i}$ meccanismi fisiologici di regolazione idroelettrolitica sono sufficienti a mantenere un corretto equilibrio, anche in caso di errata introduzione della quantità di liquidi. L'ipoalbuminemia da malnutrizione, l'iponatremia alimentare o da perdita possono tuttavia creare alterazioni dello stato di idratazione. La valutazione dello stato nutrizionale e dello stato di idratazione è importante nei pazienti con IRC. In questi pazienti l'alterazione della capacità escretiva del paziente porta, passando attraverso le tre fasi della insufficienza renale (IRC in compenso funzionale, IRC scompensata, IRC in fase uremica terminale) da una situazione di diuresi efficace, ad una di diuresi isostenurica poliurica, per poi finire in una situazione di oliguria ed anuria. Durante la fase poliurica i nefroni residui, riescono ancora ad eliminare l'eccesso di scorie azotate. In tale situazione non si ha espansione dello spazio extracellulare e lo stato di idratazione si mantiene più $o$ meno nei limiti della norma senza che si manifesti la comparsa di segni clinici oggettivi quali edemi declivi o l'instaurarsi di una ipertensione arteriosa volume dipendente. La riduzione ulteriore della massa nefronica, comporta la tendenza a

TAB. I - STATI NUTRIZIONALI da Teodori: trattato di patologia medica

\begin{tabular}{l|l}
\hline Obesità & $\begin{array}{l}\text { alterazione della composizione corporea per notevole eccesso } \\
\text { (superiore al } 30 \% \text { del peso ideale) di grasso depositato } \\
\text { nell'organismo }\end{array}$ \\
\hline Sovrappeso & peso corporeo superiore (tra il 10 e il 30\%) al peso ideale \\
\hline Magrezza & insufficienza dei depositi di tessuto adiposo \\
\hline Dimagrimento & riduzione dinamica del tessuto adiposo \\
\hline $\begin{array}{l}\text { Denutrizione } \\
\text { Malnutrizione }\end{array}$ & $\begin{array}{l}\text { riduzione dinamica della massa magra } \\
\text { deficit cronico di apporto calorico e proteico }\end{array}$ \\
\hline Cachessia & $\begin{array}{l}\text { grave deperimento generale qualitativo e quantitativo } \\
\text { generalmente progressivo }\end{array}$ \\
\hline
\end{tabular}

trattenere liquidi, che, non più rimossi, danno edemi declivi (e se non corretti con un trattamento farmacologico o sostitutivo, presto generalizzati) ed aumento della volemia con ipertensione arteriosa volume dipendente.

Lo stato nutrizionale durante IRC tende ad alterarsi con un calo ponderale per:

1) inadeguato apporto di nutrienti;

2) ipercatabolismo.

Per quanto riguarda il primo punto, il pa- 


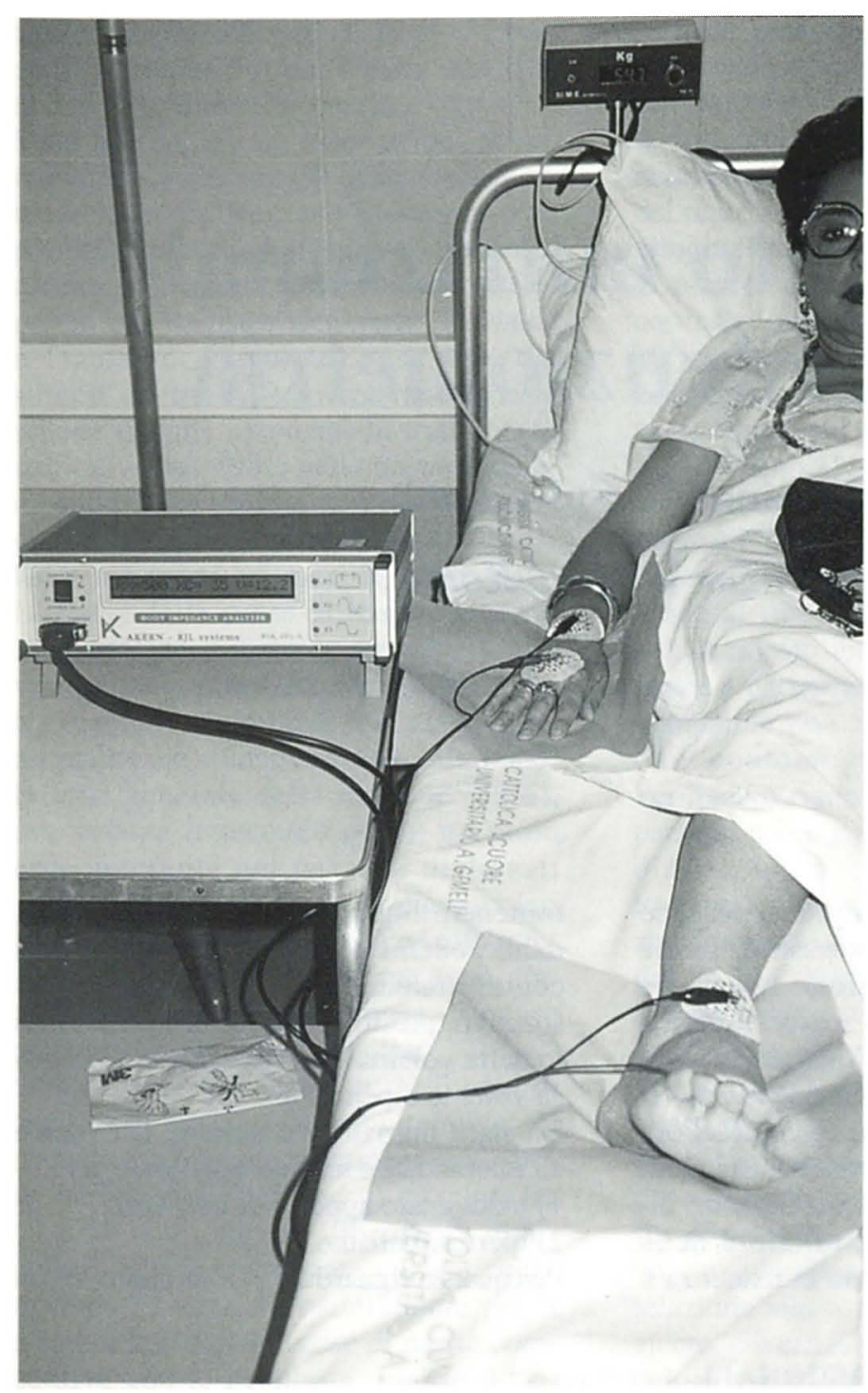

Fig. 1

ziente con IRC è un paziente che tende a non alimentarsi correttamente per il rifiuto dei cibi carnei (ciò per l'alto livello di azotemia e quindi di urea nel sangue). Inoltre non trova piacere in quello che mangia per carenza di $\mathrm{Zn}$, responsabile del senso del gusto.

La stimolazione dei processi catabolici avviene invece sia per la condizione di acidosi metabolica dell'uremico, con aumento della proteolisi muscolare e degradazione ossidativa degli aminoacidi ramificati (valina, isoleucina e soprattuto leucina) importanti per la sintesi proteica muscolare, sia per le alterazioni del metabolismo glucidico che si instaurano. Infatti, l'aumentata resistenza tissutale alla insulina porta ad una riduzione nella captazione degli aminoacidi a catena ramificata, mentre l'iperglucagonemia stimola la gluconeogenesi, ovvero la degra-

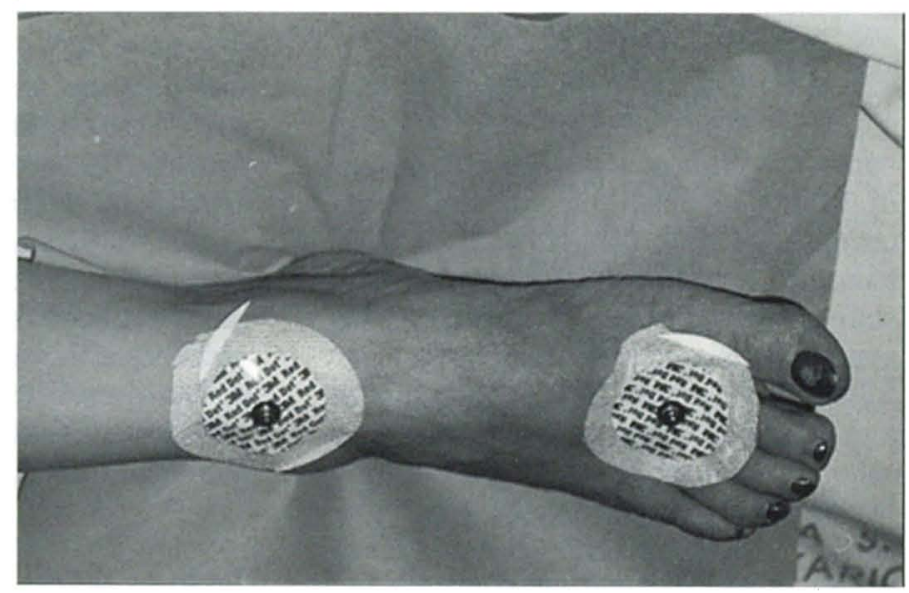

Fig. 2

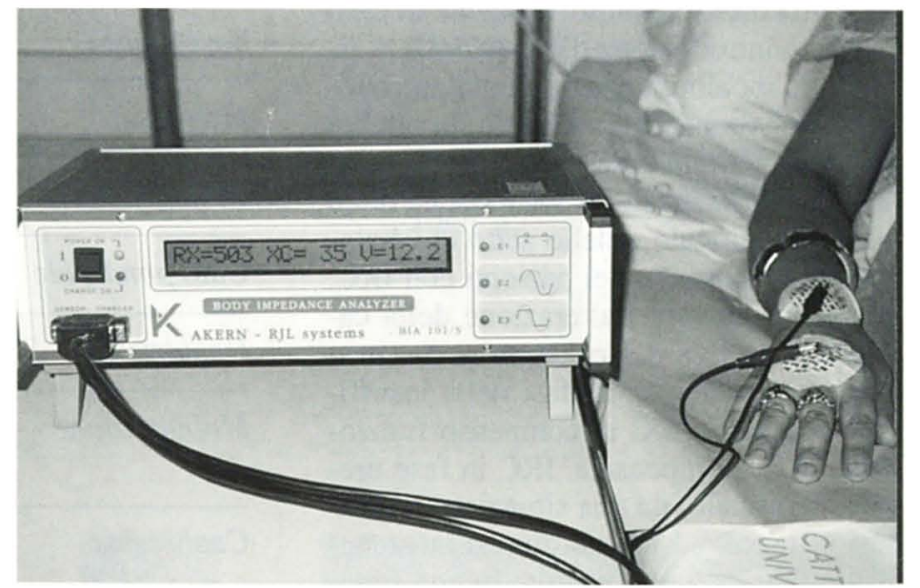

Fig. 4

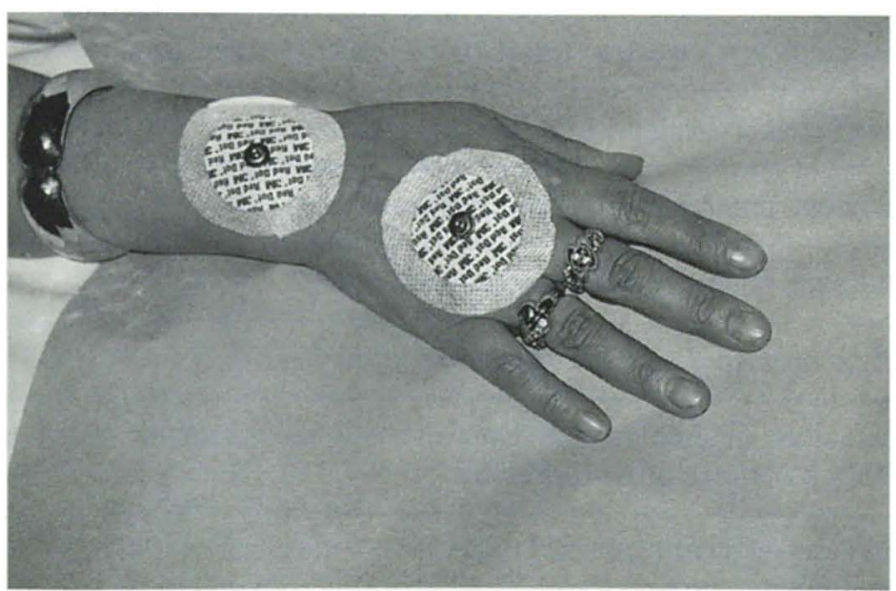

Fig. 3 
TAB. II - INDICI CORPOREI

\begin{tabular}{l|l}
\hline Broca & peso $(\mathrm{kg}) /$ altezza $(\mathrm{m})$ \\
\hline BMI & peso $(\mathrm{kg}) /$ altezza $(\mathrm{m})^{2}$ \\
\hline Rohner & peso $(\mathrm{kg}) /$ altezza $(\mathrm{m})^{3}$ \\
\hline Ponderale & peso $(\mathrm{kg})^{1 / 3} /$ altezza \\
\hline Sheldon & altezza $(\mathrm{m}) /$ peso $(\mathrm{kg})^{1 / 3}$ \\
\hline
\end{tabular}

TAB. III

\begin{tabular}{|r||r||c|}
\hline Cartella=DIA1 & Profilo Composizione Corporea & Copyright Akern \\
\hline Cognome : & Nome : & \\
Paziente numero : & Annotazioni : & \\
Età : & Resistenza : & Ohms \\
Sesso : & Reattanza : & Ohms \\
Altezza $:$ & Peso : & $\mathrm{kg}$ \\
& $\mathrm{cm}$ & FINE : Continua F10: Menù \\
F2: Cerca & &
\end{tabular}

TAB. IV

\begin{tabular}{|c||c||c||c|}
\hline Cartella Attiva & Quantità di dati & Dato Creato & Ultimo aggiornamento \\
\hline \hline DIA1 & 57 & $18-08-1994$ & $18-01-1994$ \\
\hline
\end{tabular}

\begin{tabular}{|l||}
\hline \multicolumn{1}{|c|}{ Programma Funzioni } \\
\hline \hline 1. Analisi Composizione Corporea \\
2. Analisi Statistica dei Dati \\
3. Stampare la Cartella \\
4. Creare nuova Cartella \\
5. Cancellare Cartella \\
6. Editare Cartella \\
7. Cambiare Cartella \\
8. Configurare \\
9. Selezionare esercizi \\
\hline
\end{tabular}

INVIO: Seleziona Funzione

F10:Uscire

dazione delle proteine per il loro utilizzo nella produzione di glucosio a scopi energetici. Questi fattori, assieme, portano quindi ad un impoverimento della massa muscolare.

\section{Metodiche}

È importante dunque valutare in maniera corretta lo stato idrico e nutrizionale del vazione neutronica di marker biologici alla densitometria con vasca idrostatica, dalla TOBEC all'ultrasonografia, ed altre ancora) non consentono tuttavia una valutazione istantanea dei due parametri. Inoltre, le metodiche più complesse servono molte volte a stabilire la affidabilità degli indici indiretti della composizione corporea. Con tale termine intendiamo metodiche quali la plicometria e gli indici peso/altezza.

La plicometria determina la densità corporea quantificando lo spessore del tessuto adiposo sottocutaneo valutato mediante misurazione di pliche cutanee e quindi ci permette di valutare la massa grassa (FAT) o lipidica totale, e la massa magra (FFM) o scheletrica e muscolare (tessuti magri). Le pliche normalmente studiate sono quattro: bicipitale, tricipitale, sottoscapolare e sovrailiaca; partendo dal presupposto che vi sia stretto rapporto tra spessore delle pliche e percentuale di grasso totale corporeo la somma delle quattro rilevazioni (ottenute in millimetri), permette, mediante l'ausilio di tabelle valutative di riferimento ordinate per sesso/età, di risalire alla FAT e quindi, per differenza, alla FFM. La difficoltà sta nel valutare con questi dati l'acqua corporea totale, con una metodica che può risentire di errori soggettivi, quali la non corretta presa della plica cutanea oppure l'erronea lettura della misurazione ottenuta.

Gli indici corporei impostati sui dati peso/altezza, sono indicati nella Tabella II.

Essi sono tutti ben correlati al grado di adiposità (migliore il Body Mass Index o BMI), ma tutti non permettono di differenziare un sovrappeso da un aumento di adiposità oppure da un aumento della massa muscolare oppure da una ritenzione idrosalina; richiedono quindi il supporto della valutazione clinica.

In questo panorama di incertezza diagnostica bene si inserisce la impedenziometria o BIA (Body Impedance Analyzer), metodica che permette lo studio ed il rilievo istantaneo dei compartimenti intra ed extracellulare e quindi, dell'acqua totale, della massa magra (FFM) e della massa grassa (FAT). Il punto di partenza è il rilievo di due valori: uno, denominato resistenza $(\mathrm{Rx})$, che valuta lo stato di idratazione del soggetto in esame; altro non è che la forza che si oppone al passaggio di una corrente in un corpo conduttore, quale l'acqua e gli elettroliti, presenti in larga misura nel corpo umano. L'altro valore è la reattanza $(\mathrm{Xc})$. 
TAB. V

\begin{tabular}{|l||c||c|}
\hline Cartella=DIA1 & Editare Dato & Copyright Akern \\
\hline
\end{tabular}

$\begin{array}{lllllll}\text { Cognome } & : & \text { Nome } & & \\ \text { Paziente numero } & : & \text { Annotazioni } & : & \text { interdialisi } \\ \text { Età } & : & 61 & \text { Resistenza } & : & 480 & \text { Ohms } \\ \text { Sesso } & : & \text { M } & \text { Reattanza } & : & 33 & \text { Ohms } \\ \text { Altezza } & : & 177 \mathrm{~cm} & \text { Peso } & : & 75.5 & \mathrm{~kg}\end{array}$

Vuole consultare i valori di riferimento? $\quad(\mathrm{S} / \mathrm{N})$ ?
Acqua corporea $\quad$ : 45.0 litri
Percentuale Acqua : $59.6 \%$
Peso Massa Grassa : $13.4 \mathrm{~kg}$
Massa Grassa : $17.8 \%$
Peso Massa Magra : $62.1 \mathrm{~kg}$
Massa Magra $\quad: \quad 82.2 \%$
Rapporto FFM/FM : 4.6 a 1
Densità Corporea : 1.05 g/ccm
Angolo di Fase : $\quad 3.9$
Metabolismo basale : $1815 \mathrm{kcal}$

\section{DATI SEGUENTI SI RIFERISCONO AD UN MODELLO A 3 COMPARTIMENTI}

$\mathrm{Na} / \mathrm{K}$ scambiabile : 1.56

Acqu extracell : 25.2 litri

Acqua intracell : 19.8 litri
Peso della MASSA CELLULARE: $23.7 \mathrm{~kg}$

Percent acqua extracellulare : $56.0 \%$

Percent massa cellulare $\quad: 31.5 \%$

FINE: Continua

TAB. VI

Parametri Ottimali di Riferimento

(secondo Moore, Olesen, Mc Murray, Parker, Ball, Boyden)

$\begin{array}{lll}\text { Massa } & \text { Cellulare } & (\% \text { del peso }) \\ \text { età } & \text { maschi } & \text { femmine } \\ <30 & >45 \% & >42 \% \\ <50 & >43 \% & >40 \% \\ <70 & >40 \% & >38 \%\end{array}$

Acqua Totale (\% del peso)

età maschi femmine

$<30 \quad 62 \% \quad 46 \%$

$<50 \quad 55 \% \quad 45 \%$

$<70 \quad 50 \% \quad 43 \%$

Massa Grassa (\% del peso)

età maschi femmine

$<30 \quad 15-18 \% \quad 16-20 \%$

$<50 \quad 18-20 \% \quad 22-26 \%$

$<70 \quad 20-22 \% \quad 28-30 \%$

Peso Scheletrico (\% della FFM) maschi

$11 \%$

femmine

$10 \%$
$\mathrm{Na} / \mathrm{K}$ Scambiabile (rapporto)

età maschi femmine

$\begin{array}{lll}<30 & 0.8 & 0.9\end{array}$

$\begin{array}{lll}<50 & 0.9 & 1.0\end{array}$

$<70 \quad 1.0 \quad 1.1$

Acqua Extracellulare ( $\%$ della TBW)

età maschi femmine

$<30 \quad 43-45 \% \quad 43-47 \%$

$<50 \quad 46-49 \% \quad 48-50 \%$

$<70 \quad 50-52 \% \quad 52-55 \%$

\begin{tabular}{|c|c|c|}
\hline \multicolumn{3}{|c|}{ Angolo di Fase } \\
\hline età & maschi & femmi \\
\hline$<30$ & $6-8$ & $6-7$ \\
\hline$<50$ & $5.5-6$ & $5-6$ \\
\hline$<70$ & $5.5-6$ & $4.8-5.2$ \\
\hline
\end{tabular}

Volume Ematico (\% della FFM)

età maschi femmine

$<30 \quad 9.3 \% \quad 9.8 \%$

$<50 \quad 9.2 \% \quad 10 \%$

$<70 \quad 9.1 \% \quad 10.1 \%$ 
Indica la massa cellulare del soggetto ed è la forza che si oppone al passaggio di una corrente in un condensatore. Intendiamo con questa parola due strati conduttivi separati da uno non conduttivo: nel corpo umano condensatori sono tutte le cellule, costituite a livello della membrana basale (MB) da due strati proteici, conduttivi, separati da uno strato lipidico, non conduttivo.

La misurazione viene effettuata in condizioni di riposo, con il paziente supino sul letto (Fig. 1), le braccia divaricate di $45^{\circ}$ rispetto al tronco e le gambe anch'esse divaricate di $45^{\circ}$. Si procede a detergere bene le parti dove verranno posizionati gli elettrodi con soluzione alcolica per sgrassare la pelle ed abbassare le resistenze cutanee. Si applicano quindi gli elettrodi: due in posizione distale a livello della base delle dita del piede e della mano omolateralmente; altri due in posizione prossimale, sulla bisettrice della articolazione radio-carpica e tibio-tarsica (Figg. 2, 3).

Quindi si posizionano i morsetti collegati allo strumento: i due rossi si collegano agli elettrodi distali, che vengono da adesso chiamati esploratori, mentre $\mathrm{i}$ due neri si collegano in posizione prossimale e vengono chiamati rilevatori. Attraverso i morsetti viene inviata una corrente ad intensità e frequenza nota (0:8 mA e 50khz) che entra nel corpo dagli elettrodi esploratori ed esce da quelli rilevatori. Il BIA "legge" la sfasatura o impedenza della corrente nell'attraversare il corpo e visualizza due valori numerici di resistenza e reattanza (l'unità di misura è l'Ohm) (Fig. 4).

Tali valori vengono inviati ad un elaboratore elettronico che, con l'ausilio di un programma di software (Tab. III), li acquisisce automaticamente e li elabora con i parametri inseriti dall'operatore, quali sesso, età, altezza e peso (Tab. IV). Il risultato è una stima tricompartimentale con la quantificazione, in unità di misura ed in percentuale, dell'acqua corporea totale (TBW), della massa magra (FFM), della massa grassa (FAT), dell'acqua extracellulare (ECW), dell'acqua intracellulare (ICW), del metabolismo basale (Tab. V).

Il tutto viene comparato con parametri di riferimento dati per età e per sesso (Tab. VI).

La metodica, quindi, in tempo reale e senza provocare disagio al paziente in esame, dà una stima esatta e completa di quanto da noi richiesto a livello tricompartimentale, utile per una gestione standardizzata e routinaria del paziente uremico e normale.

Il soggetto uremico in trattamento sostitutivo emodialitico, infatti, tende ad accumulare nell'intervallo interdialitico un certo quantitativo di acqua, sia libera che legata (agli alimenti assunti con la dieta). Questa acqua, non esistendo più le condizioni fisiologiche che permettono una diuresi efficace, tende ad accumularsi nell'organismo dando sia edemi declivi (semeiologicamente valutabili alla digitopressione con il segno della fòvea), che accumulo a livello delle basi polmonari (semeiologiamente evidenziabili con il rilievo oggettivo di rantoli crepitanti a piccole bolle e soggettivo di dispnea prevalentemente notturna, per il clinostatismo).

Il rilievo semeiologico, per quanto venga in soccorso nella valutazione dello stato di idratazione del paziente, è però tardivo, in quanto permette la correzione idrica in eccesso (mediante aggiustamenti del peso corporeo) soltanto dopo che si sono venuti a verificare quadri clinici da sovraccarico idrico, e molte volte a causa di errata stima del peso corporeo nei trattamenti emodialitici precedenti.

Importante è quindi in questo caso una corretta valutazione del peso corporeo del paziente, intesa come "peso secco" ovvero peso con il quale il paziente a fine dialisi termina il trattamento sostitutivo in corretto stato di idratazione e in condizioni di benessere fisico. Tale termine sta ad indicare l'assenza di disturbi soggettivi ed oggettivi che alterino le condizioni di vita del paziente.

L'impiego del BIA permette, con la rilevazione dei valori di resistenza e reattanza durante il trattamento emodialitico, di verificare il raggiungimento dell'uguaglianza o equilibrio fra i due valori suddetti, resistenza e reattanza moltiplicato per un fattore " 10 " (costante per il soggeto nefropatico). Tale equilibrio viene accettato come indice di valutazione del peso secco, e permette in tal modo di effettuare un corretto trattamento emodialitico terminando in uno stato di idratazione ottimale.

\section{Conclusioni}

L'impedenziometria è un esame semplice, di facile realizzazione che permette di valutare lo stato nutrizionale e di idratazione del paziente uremico. In particolare è utile nell'aggiustamento del "peso secco" nei soggetti con instabilità pressoria o che vadano incontro a rapide modificazioni dello stato di idratazione in rapporto a modificazioni dello stato nutrizionale.

\section{BIBLIOGRAFIA}

1. Mandolfo S. Valutazione nutrizionale nel paziente emodializzato: ruolo della plicometria. Dialisi Oggi 1988; 24: 19-24.

2. Mandolfo S, Imbasciati E. Bioimpedenziometria, antropometria e cinetica: confronto e ripetibilità. Giorn It Nefrol 1993; 2: 111-5.

3. Piasioli S, Petrosino L, Cavallini L, Zambello A, Cesaro A, Fazion S. Lo stato nutrizionale dei dializzati: valutazione prospettica. In: Atti del $30^{\circ}$ Congresso della Società Italiana di Nefrologia. Venezia 1989: 327-32.

4. Kouw PM, Olthof GC, Ter Wee PM, et al. Assessment of postdialysis dry-weight: an application of the conductivity measurement method. Kidney Int 1992; 41: 440-4.

5. Segal KR, Burastero S, Chun A, Coronel P, Pierson RN, Wang J. Estimation of extracellular and total body water by multiple frequency bioelectrical impedance measurement. Am J Clin Nutr 1991; 54: 26-9. 
Per

NUOVE

sottoscrizioni e

RINNOVI
Cedola d'ordine per

Giornale di tecniche nefrologiche e dialitiche

4 numeri anno

$\square$ Mettere in corso regolare abbonamento

(L. 90.000/anno) (\$90.000)

Please add $\$ 30.00$ for

postage outside Europe

Pagamento con Carta di Credito*

Carta SI - Visa - Eurocard - Mastercard

* Carta di Credito No

Data di scadenza

Data di nascita

Firma

(L'ordine con Carta di Credito non è valido senza firma)

(BNL-Banca Nazionale del Lavoro Ag. 5 - Milano)

Allego ricevuta di C.C. postale N. 17447202 intestato Wichtig Editore (only for Italy)

Bonifico bancario C/C 8622

\section{Cognome}

Nome

Via n.

Cap. Città

da fatturare a

(Specificare P. IVA/C.F.)

II prepagamento è indispensabile per dare corso all'abbonamento

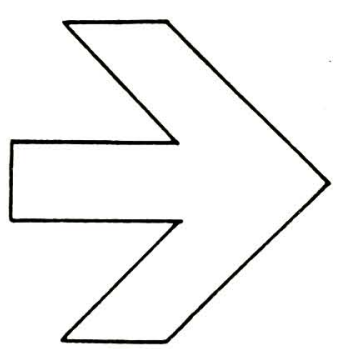

Tagliare, mettere in una busta

e spedire a

Wichtig Editore

Via Friuli, 72

20135 Milano 